Según los resultados del análisis molecular de las muestras, se encontró ADN de L. (Viannia) en 9,1\% de las muestras de mosquitos Lu. neivai estudiados. La proporción de mosquitos infectados fue de $0,9 \%$ y dichos mosquitos se capturaron en zonas de bosques primarios y secundarios, así como en áreas peridomésticas de la región de mayor incidencia de LCA en el país. En estudios previos no se encontraron parásitos en ninguno de los 3341 mosquitos hembras de la especie $L u$. neivai, como tampoco en los 94 de la especie $L u$. migonei examinados microscópicamente por otros autores, a pesar de que se recolectaron en un área con leishmaniasis hiperendémica.

Este es el primer informe publicado sobre la infección natural de mosquitos en Argentina. La aplicación de la técnica de RCP-hibridación de ácidos nucleicos permitió determinar que el parásito que circula en el noroeste argentino y que posiblemente es el agente causal de la leishmaniasis en esa región pertenece al subgénero Leishmania (Viannia). Estos resultados contribuyeron también a establecer la participación de Lu. neivai y Lu. migonei en la transmisión de esta infección. (Córdoba-Lanús E, et al. Natural infection of Lutzomyia neivai with Leishmania spp. in northwestern Argentina. Acta Trop. 2006;98(1):1-5.)

\section{Relación entre la situación socioeconómica y la obesidad en países de diferente nivel de desarrollo}

El estadio de desarrollo social y económico puede influir en la relación entre la situación socioeconómica (SSE) y la obesidad y en la forma en que esta se manifiesta en los diferentes países. Recientemente Fezeu y colaboradores (Fezeu L, Minkoulou E, Balkau B, et al. Association between socio-economic status and adiposity in urban Cameroon. Int J Epidemiol. 2006;35:105-11) examinaron la relación entre la SSE y la adiposidad en un estudio transversal de base poblacional realizado en una zona urbana de Camerún. Los autores encontraron una relación directa entre la SSE y la obesidad, a diferencia de lo encontrado por otros autores en países desarrollados. Por la importancia del tema, en este artículo se discute la forma de interpretar esos resultados a la luz del creciente e importante problema de la obesidad.

Un aspecto metodológico fundamental en cualquier estudio sobre SSE y obesidad es la conveniencia de ajustar algunos factores. Sin embargo, el consumo y el gasto energético realmente funcionan como mediadores, por lo que no se deben ajustar en este tipo de estudios. La actividad física está estrechamente relacionada con el nivel de gasto energé- tico y tanto el tiempo libre como el tiempo dedicado a alguna actividad física influyen en ello. Por lo tanto, el tratamiento cuidadoso del tiempo dedicado a actividades físicas y del tiempo libre es crucial para lograr resultados sin sesgos en este tipo de estudio.

En los países desarrollados de Europa y de América se ha encontrado - con raras excepcionesuna asociación inversa entre la SSE y la obesidad, independientemente de la forma de evaluar la SSE, ya sea en función del nivel educacional, los ingresos o la ocupación laboral. No obstante, esa asociación ha resultado directa en la mayoría de los países en estadios más tempranos de desarrollo.

Esa asociación directa encontrada entre la SSE y la obesidad en estudios transversales como el realizado en Camerún no permite establecer una relación causal que implique que la obesidad tienda a reducir el SSE o que el aumento de la obesidad y la disminución de la SSE puedan tener causas comunes. Por el contrario, esa asociación directa entre la SSE y la obesidad calculada a partir del confort del hogar y la adiposidad parece apoyar la hipótesis de que la transición en el desarrollo socioeconómico de una comunidad va acompañada de cambios en el estilo de vida (patrones alimentario y de actividad física, entre otros) que pueden explicar esa asociación.

Esa transición en los hábitos alimentarios y en la práctica de actividad física conduce a una mayor prevalencia temporal de la obesidad. Estudios recientes informan que en los primeros estadios de desarrollo económico y social de cualquier país, la carga de la obesidad pasa rápidamente a los segmentos de la población de menos recursos. Esto puede explicarse porque desaparecen poco a poco la escasez de alimentos y el gran gasto energético de los más pobres, mientras que las personas con mejor SSE adquieren alimentos más apropiados y conservan valores culturales que llevan a presumir de un cuerpo esbelto como símbolo de pertenecer a un segmento privilegiado de la población. En otras palabras, con el desarrollo socioeconómico desaparecen los factores que protegen a los pobres contra la obesidad, mientras que se mantienen y fortalecen los que protegen a las clases de mejor SSE.

De todo lo expresado se concluye que la asociación directa entre la SSE y la obesidad observada en los países en desarrollo no debe interpretarse como un indicador de que los segmentos más desfavorecidos gozan de mejor salud. En los países que comienzan a experimentar un acelerado desarrollo socioeconómico se deben tomar medidas para evitar la obesidad y los trastornos asociados con ella desde mucho antes de que comiencen a incrementarse las tasas de obesidad en la población. (Song YM. Commentary: varying relation of socioeconomic status with obesity between countries at diffe- 
rent stages of development. Int J Epidemiol. 2006; 35(1):112-3.)

\section{Revisión sistemática sobre el efecto de Plasmodium falciparum en la función cognoscitiva}

La infección por Plasmodium falciparum afecta a alrededor de 500 millones de personas en el mundo y causa 2,7 millones de muertes cada año. Esta es una de las principales especies causantes de malaria y al parecer puede causar daños al sistema nervioso central. Cada día gana más respaldo la hipótesis de que la infección grave por $P$. falciparum daña de manera más o menos persistente la capacidad cognoscitiva del enfermo, aunque no se sabe en qué medida esto pueda ocurrir cuando la enfermedad es menos grave. Este cuadro se hace aun más confuso por los diferentes criterios empleados para clasificar la gravedad de la enfermedad y por los diversos factores de riesgo que se han propuesto.

Los autores de este trabajo realizaron una revisión sistemática de los efectos de $P$. falciparum sobre la cognición, en particular la relación entre el parásito y la función cognoscitiva y las posibles diferencias en el deterioro neurológico y cognoscitivo en los niños y en los adultos.

La búsqueda abarcó todo lo publicado hasta agosto de 2005 y referido en MEDLINE (desde 1966), EMBASE (desde 1980) y PsycINFO (desde 1987). Los manuscritos se identificaron mediante la combinación de términos y palabras clave o por los títulos temáticos. Se revisaron las referencias bibliográficas de los artículos encontrados y las tesis de doctorado. Finalmente se analizó el texto completo de 25 documentos, de los cuales se descartaron 7 por no emplear pruebas estandarizadas para la población estudiada o controles adecuados o por no diferenciar claramente los resultados en niños y adultos.

En los niños, las formas graves de malaria estuvieron asociadas con un mayor grado de daño neurológico y cognoscitivo. En los adultos se encontraron deficiencias cognoscitivas relacionadas con la atención, la memoria y el lenguaje, pero no con la percepción visual o espacial o las funciones ejecutivas.

Aún no se conoce el mecanismo exacto mediante el cual el parásito $P$. falciparum causa el daño neurológico o cognoscitivo. La malaria puede influir negativamente en la cognición de manera indirecta, a través de una mala nutrición, la ausencia a la escuela o entorpeciendo el desarrollo psicosocial del enfermo. Sin embargo, hay pruebas contundentes de que la malaria cerebral causa trastornos como resultado de los daños directos que ocasiona en el cerebro. Trastornos específicos, como la disfunción del hipocampo y la lesión de la materia blanca subcorti- cal, pueden provocar alteraciones de la memoria y del lenguaje. Un estudio señaló la posibilidad de que la malaria cerebral provoque daños estructurales en la materia blanca del cerebro. Según otra hipótesis, las neurotoxinas liberadas por los glóbulos rojos infectados pueden dañar las áreas corticales del cerebro y así menoscabar las funciones cognoscitivas del enfermo. Como la comprensión del lenguaje, el aprendizaje y el razonamiento se ven mediados por la memoria, es posible que el daño neurológico y cognoscitivo provocado por la malaria esté relacionado con esta zona del cerebro.

A pesar de algunas limitaciones de este estudio, los resultados indican que la malaria puede provocar deficiencias en la atención, la memoria, las habilidades visuales y espaciales, el lenguaje y las funciones ejecutivas, tanto a corto como a largo plazo. Estos daños pueden observarse en casos de malaria cerebral por $P$. falciparum, pero también en formas menos graves de la enfermedad. La información disponible indica que es posible que este parásito afecte al cerebro en su conjunto y no de forma localizada. Al parecer, la edad en que ocurre la infección y el número de reinfecciones pueden ser variables que determinan la magnitud del daño neurológico y cognoscitivo.

El hecho de que cada año se infecten más de 500 millones de personas con este parásito hace pensar que la malaria puede ser una de las causas más frecuentes de trastorno cognoscitivo en el mundo. (Kihara M, et al. The effect of Plasmodium falciparum on cognition: a systematic review. Trop Med Int Health. 2006;11(4):386.)

\section{Factores sociodemográficos y ambientales asociados con el desmedro infantil en zonas rurales de Guatemala}

El retraso en el crecimiento o desmedro está asociado con el incremento de la morbilidad y la mortalidad en los niños y se calcula que alrededor de 182 millones de niños de países en desarrollo presentan este trastorno. A pesar del carácter multifactorial de las causas del desmedro, se ha documentado el papel causal que desempeñan la desnutrición y las infecciones frecuentes durante la infancia. Las deficientes condiciones de saneamiento y de higiene en el hogar, la mala situación socioeconómica, las diarreas frecuentes y el déficit de agua potable también pueden contribuir al desmedro. La incidencia de más de uno de estos factores aumenta el riesgo de padecerlo.

$\mathrm{Al}$ menos $50 \%$ de los niños de 30 a 35 meses de edad en Guatemala presentan una relación tallaedad por debajo de la línea de corte de desmedro. Se calcula que hasta $74 \%$ de los niños menores de 5 